\title{
Compliance with Guidelines for Treatment of Staphylococcus aureus Bacteremia is Associated with Decreased Mortality in Patients Hospitalized for Community-Acquired Pneumonia with Staphylococcus aureus Bacteremia
}

\author{
Leslie Beavin ${ }^{1 *}, M D$; Vidyulata Salunkhe ${ }^{1}, M D$ MPH; Shashvin Singh ${ }^{1}, M D ;$ Ahmed Gana ${ }^{1}, M D ;$ Balaji Sekaran ${ }^{1}, M D ;$ Tella Mahder $^{1}, M P H ;$ \\ Stephen Furmanek ${ }^{1}$, MS MPH; Forest W. Arnold ${ }^{1}$, DO, MSC \\ ${ }^{1}$ Division of Infectious Diseases, School of Medicine, University of Louisville, Louisville, KY, USA \\ *leslie.beavin@louisville.edu
}

Recommended Citation: Beavin L, Salunkhe V, Singh S, et al. Compliance with guidelines for treatment of Staphylococcus aureus bacteremia is associated with decreased mortality in patients hospitalized for community-acquired pnuemonia with Staphylococcus aureus bacteremia. Univ Louisville $\mathrm{J}$ Respir Infect 2022; 6(1):Article 5. doi: 10.18297/jri/vol6/iss1/5.

\begin{abstract}
Introduction: Staphylococcus aureus bacteremia has a minimum treatment duration of two weeks, while $S$. aureus community-acquired pneumonia (CAP) treatment is at least five days. Treatment failure, persistent bacteremia, and recurrence are common among patients with community-acquired $S$. aureus bacteremia. There is conflicting information in the current Infectious Diseases Society of America (IDSA) guidelines for the treatment of $S$.aureus bacteremia patients with CAP. Therefore, the appropriate treatment duration and modality for $S$. aureus CAP with bacteremia is unclear. The objective of this study was to compare outcomes among patients with $S$. aureus CAP and bacteremia treated in compliance versus non-compliance with IDSA $S$. aureus bacteremia guidelines.
\end{abstract}

Methods: This was a secondary data analysis of the Community-Acquired Pneumonia Organization (CAPO) study database. Logistic regression was used to compare outcomes.

Results: A total of 117 patients with S. aureus CAP and bac- teremia were included in the study. Compliance with $S$. aureus bacteremia guidelines was documented in 67 patients, and non-compliance was documented in 50 patients. Compliance with IDSA $S$. aureus bacteremia guidelines resulted in a decrease in odds of re-hospitalization of $30 \%$ after adjusting for confounding variables between the compliant and non-compliant groups (adjusted odds ratio (aOR) $0.70[95 \%$ Cl 0.29-1.70]; $P=0.42$ ). The 30-day mortality for the compliant group was $6 \%$ and for the non-compliant group was $10 \%$; $P=0.576$. The 1 -year mortality for the compliant group was $19 \%$ and for the non-compliant group was $44 \% ; P=0.011$.

Conclusion: The present study demonstrated that when treated in compliance with IDSA guidelines for $S$. aureus bacteremia, there was decreased 1-year mortality for patients hospitalized for $S$. aureus CAP with bacteremia. In this case, the IDSA S. aureus bacteremia guidelines recommend treating uncomplicated $S$. aureus bacteremia with CAP for at least two weeks of antimicrobials and at least four weeks of antimicrobials for complicated $S$. aureus bacteremia with CAP.

\section{Introduction}

Staphylococcus aureus infections are associated with significant morbidity and mortality.[1] While $S$. aureus is responsible for less than $5 \%$ of microbiologically confirmed cases of community-acquired pneumonia (CAP), it is associated with an increased need for hospitalization, including intensive care unit (ICU) admission, increased length of hospital stay, and increased mortality compared to pneumococcal and other nonS. aureus causes of pneumonia.[2] Studies combining pneumonia, either acquired in the hospital or from the community, due to $S$. aureus with associated bacteremia have demonstrated mortality up to nearly $50 \%$ at 30 days.[3, 4] In addition, S. aureus bacteremia itself is often associated with other significant complications, such as intra-abdominal, iliopsoas, and epidural abscesses, as well as endovascular infections.[1]

The current Infectious Disease Society of America (IDSA) guidelines for CAP discuss a minimal treatment duration of five days.[5] A switch from intravenous to 
Figure 1. Study population.

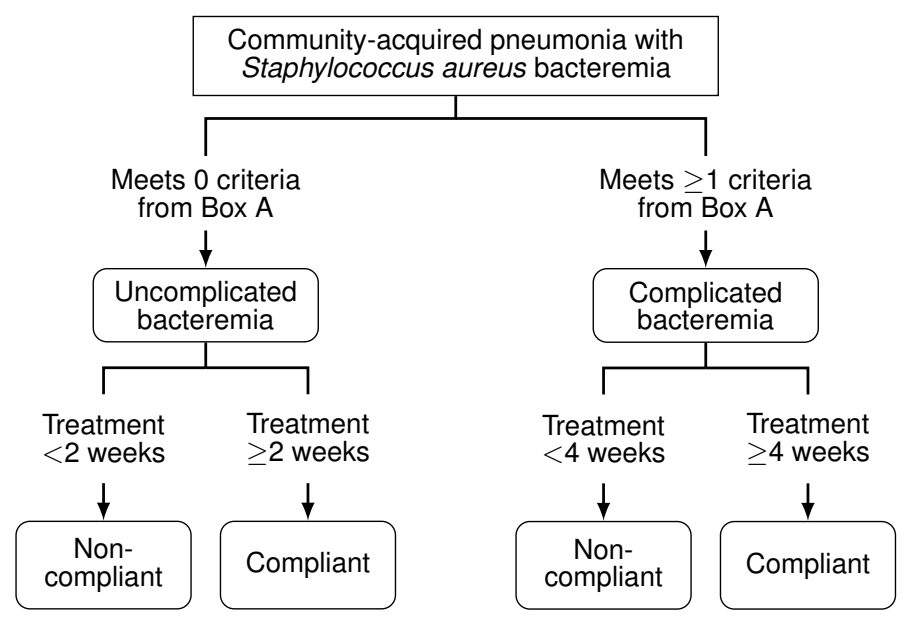

Box A. Complications of bacteremia
1. Temperature that did not drop below $100.5^{\circ} \mathrm{F}$
during the first 3 days of antimicrobial therapy.
2. Echocardiogram revealing valvular pathology or
a vegetation.
3. Prosthetic material or joints.
4. Immunocompromising condition or disease,
such as organ transplantation, active neoplastic
disease, autoimmune disease, or
immunosuppressive therapy.
5. Metastatic sites of infection with $S$. aureus.
6. Persistent bacteremia, defined as having $>1$
positive blood culture for $S$. aureus when
cultures drawn at least 30 minutes apart.

oral antimicrobial therapy is recommended when the patient is clinically stable and can tolerate and absorb oral antimicrobials. [5] However, there is no specific recommendation for $S$. aureus CAP complicated by bacteremia in the 2019 IDSA CAP guidelines. Therefore, the guidelines did not address this significant point and did not give specific recommendations on managing patients with $S$. aureus CAP complicated by bacteremia, thus being quite different from the IDSA guidelines for $S$. aureus bacteremia, which recommend a minimum treatment duration of 14 days for uncomplicated bacteremia and at least 4-6 weeks of intravenous antimicrobial therapy for complicated bacteremia.[6] Given the conflicting information in the current IDSA guidelines, the appropriate treatment duration and modality for $S$. aureus CAP with associated bacteremia remains unclear. A recent opinion piece addressed this conflict in treatment recommendations and suggested that in cases of $S$. aureus CAP associated with bacteremia, bacteremia itself should be the primary diagnosis guiding treatment.[7] However, more definitive data are needed to support this recommendation. The objective of this study was to compare outcomes among patients with $S$. aureus CAP with bacteremia treated in compliance versus non-compliance with IDSA $S$. aureus bacteremia guidelines.

\section{Methods}

\section{Study design and subjects}

The study design was a secondary data analysis of the Community-Acquired Pneumonia Organization (CAPO) study database. CAPO is a retrospective observational study of hospitalized adults with CAP in 130 institutions in 28 countries. Patients included in the study were hospitalized at four specific Louisville hospitals with CAP and at least one blood culture pos- itive for S. aureus. Continuous patients were included from December 2012 to March 2018. Patients were excluded if they were pregnant, had a history of intravenous drug use, were younger than 18 years of age, had antimicrobials or medical care withdrawn during the study, did not have follow-up blood cultures, or left against medical advice. This study was approved by the University of Louisville Institutional Review Board (IRB number 11.0613).

\section{Definitions}

A patient was defined as having CAP when the following two criteria were met: 1) presence of a new pulmonary infiltrate on chest radiograph or chest computed tomography scan at the time of hospitalization and 2) at least one of the following: a) new cough or increased cough or sputum production, b) fever $>37.8^{\circ} \mathrm{C}$ $\left(100.0^{\circ} \mathrm{F}\right)$ or hypothermia $\left.<35.6^{\circ} \mathrm{C}\left(96.0^{\circ} \mathrm{F}\right), \mathrm{c}\right)$ changes in leukocyte count (leukocytosis: $>11,000$ cells $/ \mathrm{mL}$; left shift: $>10 \%$ band forms $/ \mathrm{mL}$; or leukopenia: $<4000$ cells $/ \mathrm{mL}$ ).

A patient with CAP with S. aureus bacteremia was defined as a patient admitted to the hospital for CAP in whom $S$. aureus was isolated from a blood culture. Patients with hospital-acquired pneumonia (HAP) were omitted.

A patient was defined as being compliant or noncompliant with the IDSA S. aureus guidelines based on having complicated or uncomplicated bacteremia and on the duration of therapy (see Figure 1). A patient was defined as having complicated bacteremia when one or more of the following was present: 1) a temperature that did not drop below $100.5^{\circ} \mathrm{F}$ during the first three days of antimicrobial therapy; 2) an echocardiogram revealing valvular pathology or vegetation; 3 ) prosthetic material or joints; 4) an immunocompromis- 
Table 1. Patient Characteristics of Staphylococcal aureus CAP Bacteremia Patients as Non-Compliant and Compliant with IDSA S. aureus Bacteremia Guidelines.

\begin{tabular}{|c|c|c|c|c|}
\hline & $\begin{array}{l}\text { Non-compliant* } \\
(n=50)\end{array}$ & $\begin{array}{l}\text { Compliant* } \\
(n=67)\end{array}$ & $P$ & Missing (\%) \\
\hline $\begin{array}{l}\text { Demographics } \\
\text { Age } \\
\text { Male sex } \\
\text { African American }\end{array}$ & $\begin{array}{c}58[49,72] \\
23(46) \\
2(4)\end{array}$ & $\begin{array}{c}59[46,70] \\
32(48) \\
2(3)\end{array}$ & $\begin{array}{l}0.637 \\
0.999 \\
0.441\end{array}$ & $\begin{array}{l}0 \\
0 \\
0\end{array}$ \\
\hline $\begin{array}{l}\text { Laboratory findings } \\
\text { Heart rate (beats/minute) } \\
\text { Respiratory rate (breaths/minute) } \\
\text { Systolic blood pressure }(\mathrm{mmHg}) \\
\text { Diastolic blood pressure }(\mathrm{mmHg}) \\
{\mathrm{Temperature}\left({ }^{\circ} \mathrm{C}\right)} \\
\mathrm{PaO}_{2}(\mathrm{~mm} \mathrm{Hg}) \\
\mathrm{Blood}_{\text {Srea nitrogen }(\mathrm{mg} / \mathrm{dL})} \\
\text { Serum glucose }(\mathrm{mg} / \mathrm{dL}) \\
\text { Serum sodium }(\mathrm{mEq} / \mathrm{L}) \\
\text { Hematocrit }\end{array}$ & $\begin{array}{c}114[104,129] \\
24[20,29] \\
106[88,124] \\
50[42,59] \\
38[37,39] \\
66[56,86] \\
21[17,41] \\
140[111,189] \\
138[134,141] \\
33[29,36]\end{array}$ & $\begin{array}{c}112[100,125] \\
24[20,30] \\
104[92,119] \\
54[45,61] \\
38[37,39] \\
88[66,103] \\
29[16,36] \\
143[118,199] \\
135[132,139] \\
32[28,36]\end{array}$ & $\begin{array}{l}0.236 \\
0.606 \\
0.851 \\
0.470 \\
0.491 \\
0.043 \\
0.517 \\
0.550 \\
0.081 \\
0.589\end{array}$ & $\begin{array}{c}0 \\
0 \\
0 \\
0 \\
0 \\
64.1 \\
0 \\
0.9 \\
0 \\
0\end{array}$ \\
\hline $\begin{array}{l}\text { Medical and social history } \\
\text { Altered mental status } \\
\text { Alcohol abuse } \\
\text { Congestive heart failure } \\
\text { COPD } \\
\text { Diabetes mellitus type II } \\
\text { Neoplastic disease } \\
\text { Renal disease } \\
\text { Cerebrovascular disease } \\
\text { Renal failure } \\
\text { Suspicion of aspiration } \\
\text { Nursing home resident } \\
\text { Hospitalized }>2 \text { days in the prior } 90 \text { days } \\
\text { Home infusion therapy in the prior } 30 \text { days } \\
\text { Chronic dialysis in the prior } 30 \text { days } \\
\text { Home wound care } \\
\text { Family history of coronary artery disease } \\
\text { Coronary artery disease } \\
\text { Essential arterial hypertension } \\
\text { Hyperlipidemia } \\
\text { Prior myocardial infarction } \\
\text { Prior PTCA/CABG }\end{array}$ & $\begin{array}{l}16(32) \\
3(6) \\
13(26) \\
18(36) \\
20(40) \\
8(16) \\
13(26) \\
12(24) \\
9(18) \\
3(6) \\
8(16) \\
21(42) \\
8(16) \\
4(8) \\
2(4) \\
14(28) \\
13(26) \\
32(64) \\
18(36) \\
10(20) \\
7(14)\end{array}$ & $\begin{array}{l}16(24) \\
2(3) \\
15(22) \\
14(21) \\
29(43) \\
5(7) \\
21(31) \\
6(9) \\
10(15) \\
3(4) \\
8(12) \\
28(42) \\
4(6) \\
7(10) \\
5(7) \\
17(25) \\
17(25) \\
40(60) \\
27(40) \\
8(12) \\
11(16)\end{array}$ & $\begin{array}{c}0.444 \\
0.737 \\
0.815 \\
0.109 \\
0.868 \\
0.248 \\
0.672 \\
0.049 \\
0.847 \\
1 \\
0.719 \\
1 \\
0.144 \\
0.898 \\
0.699 \\
0.915 \\
1 \\
0.779 \\
0.779 \\
0.349 \\
0.921\end{array}$ & $\begin{array}{l}0 \\
0 \\
0 \\
0 \\
0 \\
0 \\
0 \\
0 \\
0 \\
0 \\
0 \\
0 \\
0 \\
0 \\
0 \\
0 \\
0 \\
0 \\
0 \\
0 \\
0\end{array}$ \\
\hline $\begin{array}{l}\text { Severity of disease } \\
\text { Ventilatory support received } \\
\text { Vasopressors received } \\
\text { Direct admission to the ICU }\end{array}$ & $\begin{array}{c}10(20) \\
7(14) \\
16(32)\end{array}$ & $\begin{aligned} 14 & (21) \\
6 & (9) \\
22 & (33)\end{aligned}$ & $\begin{array}{c}1 \\
0.594 \\
1\end{array}$ & $\begin{array}{c}0 \\
0.9 \\
0\end{array}$ \\
\hline $\begin{array}{l}\text { Pneumonia severity index } \\
\text { Risk class } \\
\text { Risk Class I } \\
\text { Risk Class II } \\
\text { Risk Class III } \\
\text { Risk Class IV } \\
\text { Risk Class V } \\
\text { Pneumonia severity index }\end{array}$ & $\begin{array}{c}4(8) \\
9(18) \\
6(12) \\
12(24) \\
19(38) \\
116[70,150]\end{array}$ & $\begin{array}{c}4(6) \\
12(18) \\
9(13) \\
23(34) \\
19(28) \\
105[74,138]\end{array}$ & 0.405 & \\
\hline
\end{tabular}

Abbreviations: ABX, antibiotics; COPD, chronic obstructive pulmonary disease; ICU, intensive care unit; IDSA, Infectious Diseases Society of America; IQR, interquartile range; PTCA/CABG, percutaneous transluminal coronary angioplasty/coronary artery bypass grafting.

${ }^{*}$ Compliance defined as following IDSA Guideline reocmmended antimicrobial treatment. Data summarized as $n(\%)$ or median [IQR]. 
Table 2. Reasons for readmission within one year among patients with Staphylococcus aureus community-acquired pneumonia and bacteremia.

\begin{tabular}{lc}
\hline Diagnosis & $n(\%)$ \\
\hline Bacteremia & $16(40)$ \\
Sepsis/septic shock & $6(15)$ \\
Pneumonia & $5(13)$ \\
Endocarditis & $3(8)$ \\
Line/port/vascular graft infection & $3(8)$ \\
Osteomyelitis & $2(5)$ \\
Cellulitis & $2(5)$ \\
Septic arthritis & $1(3)$ \\
Abscess & $1(3)$ \\
\hline
\end{tabular}

ing condition or disease, such as organ transplantation, active neoplastic disease, autoimmune disease, or immunosuppressive therapy; 5) metastatic sites of infection with S. aureus; or 6) persistent bacteremia, defined as having more than one blood culture positive for $S$. aureus when cultures were drawn at least 30 minutes apart. A patient who did not meet the criteria for complicated bacteremia was considered to have uncomplicated bacteremia.[7]

Patients were divided into two groups depending on the treatment received during hospitalization. Patients were included in the compliant group if antimicrobials were prescribed according to the IDSA guidelines for $S$. aureus bacteremia. Patients with uncomplicated bacteremia should receive two weeks of an intravenous antimicrobial. Any patient presenting with complicated bacteremia should receive at least four weeks of intravenous antimicrobial treatment. Patients with a shorter duration of therapy were included in the noncompliant group.

Outcomes evaluated were time to negative follow-up blood cultures, re-hospitalization within one year due to complications of S. aureus bacteremia, and 30-day, 6month, and 1-year mortality. Death was considered as all-cause mortality. Readmission was defined as any subsequent admission within one year where $S$. aureus was isolated in culture and learned from the fluid. Echocardiogram results were collected from medical records to confirm valvular pathology or the presence of a vegetation.

\section{Statistical analysis}

Continuous variables were represented as medians and interquartile ranges. Categorical variables were represented as frequencies and percentages. Baseline categorical variables were compared using Chi-squared tests of independence, and continuous patient variables were compared using Mann-Whitney U tests. After adjusting for history of cerebrovascular disease and pneumonia severity index, logistic regression was used to compare mortality and readmission. Time to negative blood culture, readmission, and death were ana- lyzed using Kaplan-Meier curves and compared using the log-rank test. A $P$-value less than 0.05 was considered significant.

\section{Results}

A total of 117 patients with S. aureus CAP and bacteremia met inclusion criteria. Compliance with $S$. aureus bacteremia guidelines was documented in $67 \mathrm{pa}-$ tients, and non-compliance was documented in 50 patients. Patient characteristics are shown in Table 1. S. aureus-related re-hospitalizations occurred in $23 \%$ of patients overall within one year. $21 \%$ versus $26 \%$ of patients were re-hospitalized within one year in the compliant and non-compliant groups, respectively. Reasons for readmission within one year among patients with $S$. aureus community-acquired pneumonia and bacteremia are shown in Table 2 . Treatment compliance with IDSA guidelines for $S$. aureus bacteremia did not significantly influence re-hospitalization for either group; (adjusted odds ratio [aOR] 0.70 [95\% CI: 0.291.70]; $P=0.42$; Figure 2).

\section{Mortality}

The 30-day mortality for the entire population was $7.7 \%$. The 30-day mortality was $6 \%$ for the compliant group, and $10 \%$ for the non-compliant group. Adjusting for potential confounding variables, the difference in 30-day mortality between the compliant and noncompliant groups was not statistically significant (aOR 0.67 [95\% CI 0.15-2.83]; $P=0.576$ ). (Table 3)

The 6-month mortality for the total population was $24 \%$. For the compliant group, the 6-month mortality was $18 \%$, and for the non-compliant group, it was $32 \%$. Adjusting for potential confounding variables, the difference in 6-month mortality between the compliant and non-compliant groups was not statistically significant (aOR 0.51 [95\% CI 0.19-1.36]; $P=0.178$ ). (Table 3)

The 1-year mortality for the total population was 30\%. For the compliant group, the 1-year mortality was 19\%, and for the non-compliant group, it was $44 \%$. Adjusting for potential confounding variables, the compliant 


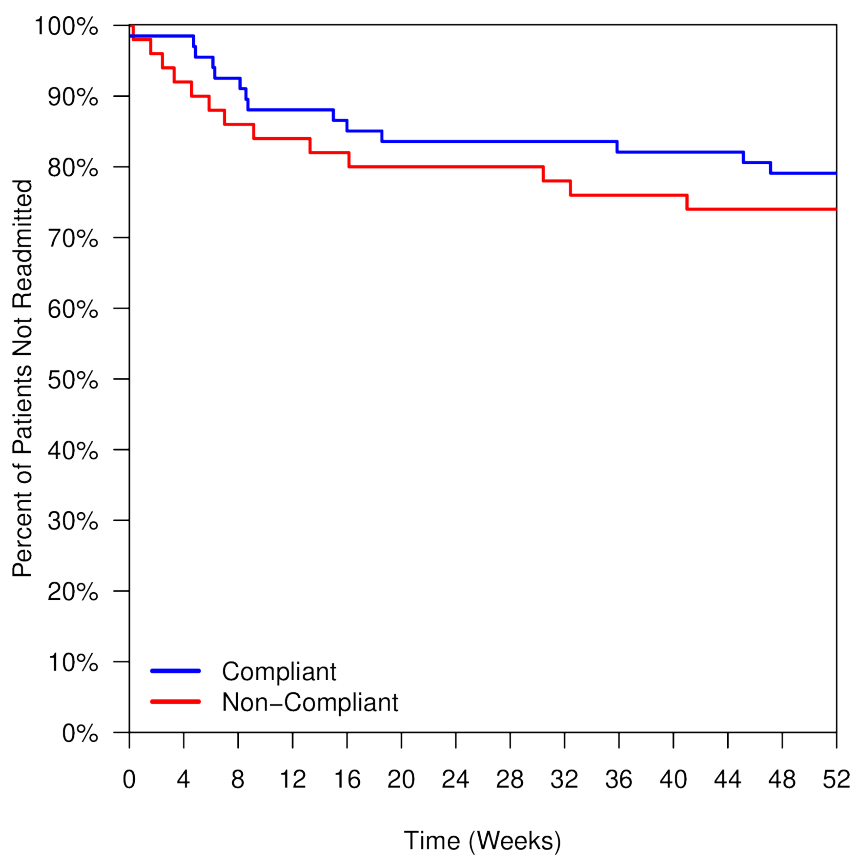

Figure 2. Kaplan-Meier curve for time to readmission within one year of hospitalization for community-acquired pneumonia with $S$. aureus bacteremia.

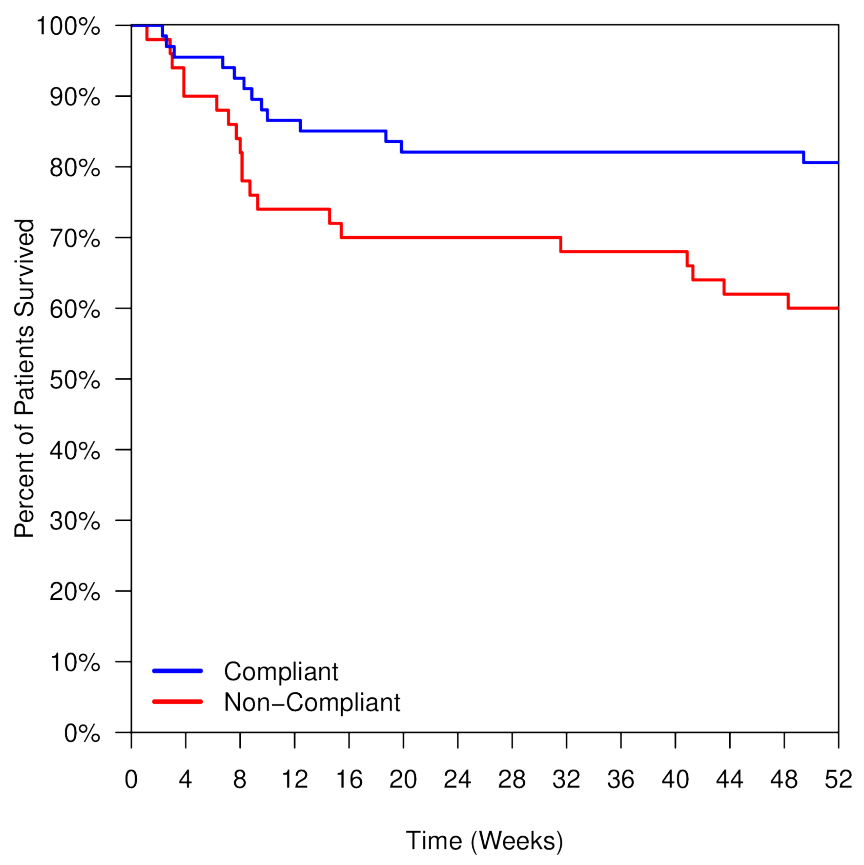

Figure 3. Kaplan-Meier curve for time to death over one year after hospitalization for community-acquired pneumonia with $S$. aureus bacteremia. group had a $69 \%$ reduction in 1-year mortality compared to the non-compliant group (aOR 0.31 [95\% CI $0.12-0.76$ ]; $P=0.011$ ). (Table 3 and Figure 3 )

Time to negative blood culture (median [interquartile range]) in the compliant group was 3.6 [1.04, 4.18] days compared to $2.4[2.34,6.02]$ days in the non-compliant group; $P=0.142$ (Table 4 and Figure 4 ).

\section{Discussion}

The present study demonstrated that patients with $S$. aureus CAP plus bacteremia, when treated according to the IDSA guidelines for $S$. aureus bacteremia (compliant) rather than the IDSA guidelines for CAP (noncompliant), had a $69 \%$ reduction in 1-year mortality (aOR 0.31 [95\% CI 0.12-0.76]; $P=0.011$ ). Suggesting a longer course of antimicrobials for CAP beyond the 5-7 days recommended per the IDSA CAP guidelines may be necessary if $S$. aureus bacteremia is also present to reduce the risk of complications. Following the IDSA guidelines for $S$. aureus bacteremia may improve longterm mortality (1 year) for patients with $S$. aureus CAP who also have $S$. aureus bacteremia.

During readmission within one year after admission for CAP with $S$. aureus bacteremia, subsequent hospitalization due to $S$. aureus infection, such as bacteremia, sepsis, endocarditis, or pneumonia (as shown in Table 2) was seen in $40 \%$ of patients. These results show that
S. aureus bacteremia with CAP is a severe infection with a higher subsequent hospitalization rate for complications. Furthermore, it is recommended to follow-up care of these patients upon hospital discharge.

The present study showed significantly lower mortality than previously described for $S$. aureus bacteremia in patients with HAP or CAP. De la Calle et al. reported 30-day total mortality for $S$. aureus bacteremia HAP and CAP as $46.9 \%$.[3] S. aureus bacteremia HAP patients may be more likely to have multiple hospitalassociated complications with subsequent higher mortality than $S$. aureus bacteremia CAP patients. However, even the 30-day mortality for $S$. aureus CAP with bacteremia has been reported as high as 37\%.[3] Again, this may reflect that most of our patients were treated with a longer duration of intravenous antibiotic therapy, complying with IDSA guidelines for S. aureus bacteremia. Thus, providing adequate treatment of the bacteremia itself is the primary predictor of outcomes for CAP patients with $S$. aureus bacteremia.

In the present study, 30-day and 6-month mortality among the compliant and non-compliant groups was not statistically significant, but the 1-year mortality was; $P=0.01$. Hence, no improvement in outcomes will be appreciated when complying or not complying with the IDSA $S$. aureus bacteremia guidelines until one year. However, this is a benefit of reporting longterm outcomes - to show that certain management is associated with higher survival. Therefore, in this case, 
Table 3. Mortality outcomes at 30 days, six months, and one year for patients hospitalized for community-acquired pneumonia with $S$. aureus bacteremia.

\begin{tabular}{lcccc}
\hline Mortality & Non-compliant & Compliant & Odds ratio $[\mathrm{Cl}]$ & $P$ \\
\hline 30-day & $5(10)$ & $4(6)$ & $0.67[0.16-2.83]$ & 0.57568 \\
6-month & $16(32)$ & $12(18)$ & $0.51[0.19-1.36]$ & 0.17778 \\
1-year & $22(44)$ & $12.7(19)$ & $0.31[0.062-0.50]$ & 0.01142 \\
\hline
\end{tabular}

Table 4. Time to negative blood culture for patients hospitalized for community-acquired pneumonia with $S$. aureus bacteremia.

\begin{tabular}{lcc}
\hline & Time to negative blood culture, median [IQR] & $P$ \\
\hline & & 0.142 \\
Compliant group $(\geq 14$-day treatment $)$ & $3.57[1.04,4.18]$ & \\
Non-compliant group $(<14$-day treatment) & $2.42[2.34,6.02]$ & \\
\hline
\end{tabular}

Abbreviations: IQR, interquartile range.

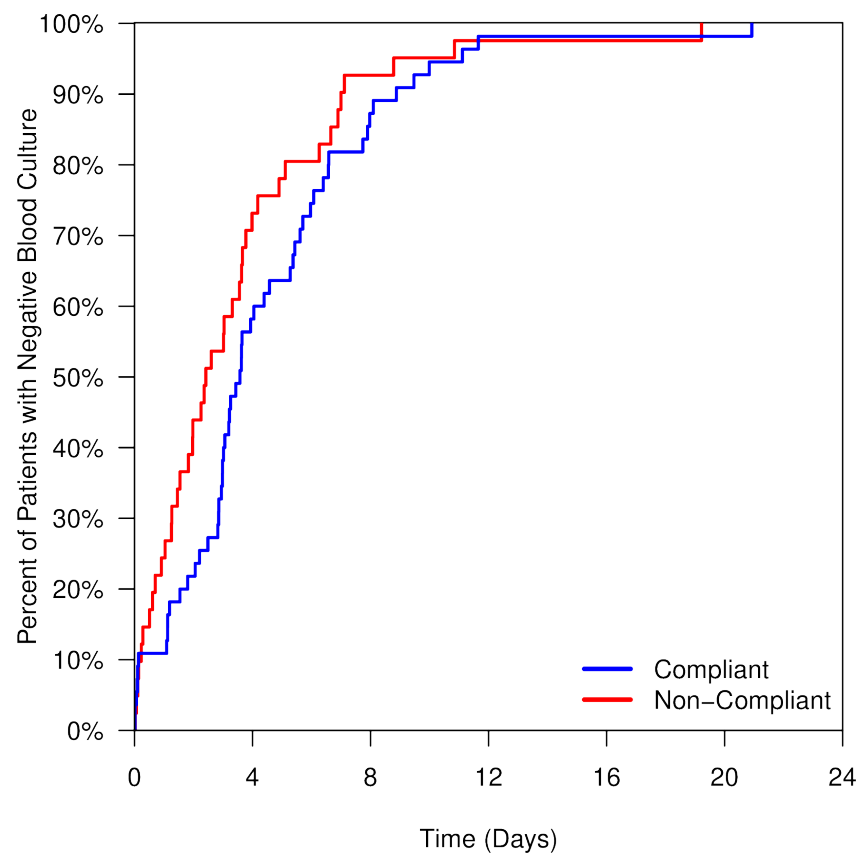

Figure 4. Kaplan-Meier curve for time to negative blood culture (in days) for patients hospitalized for community-acquired pneumonia with $S$. aureus bacteremia. 
the IDSA S. aureus bacteremia guidelines dictate treating uncomplicated $S$. aureus bacteremia with CAP for at least two weeks of antimicrobials and at least four weeks of antimicrobials for complicated S. aureus bacteremia with CAP.

A study describing long-term outcomes for all $S$. aureus infections described a $42 \%$ mortality for patients within one year of diagnosis and further reported that $43 \%$ of the patients required re-hospitalization within that year.[8] While that study did not describe the differences in treatment among the different types of $S$. aureus infections, most of the patients were treated for skin and soft tissue infections.[8] The proportion of our patients who required re-hospitalization for our entire population was 23\%. Perhaps this lower proportion was because a large proportion of the patients in our study had an infectious diseases consultation. Eighty percent of the patients included in the present study had an infectious diseases consultation, with $96 \%$ of those patients being treated in compliance with IDSA guidelines for $S$. aureus bacteremia. Infectious diseases consultation has demonstrated improved outcomes for patients with $S$. aureus bacteremia.[9] In addition, some studies found that at least $50 \%$ of patients with $S$. aureus bacteremia will develop complicated bacteremia [10], which supports appropriate management to prevent subsequent complications and hospitalizations.

Limitations of the present study primarily include its retrospective nature and relatively low number of patients (sample size of 117). Study strengths include an evaluation of long-term outcomes, including mortality as well as re-hospitalization. The two groups also had similar patient characteristics.

Overall, the present study suggests that adequate treatment of patients hospitalized for $S$. aureus CAP with bacteremia in compliance with IDSA $S$. aureus bacteremia guidelines decreases the odds of rehospitalization within the following year and improves long-term mortality. However, further research is needed to determine whether there is a difference in non-mortality outcomes, such as long-term complications.
Acknowledgements: The authors acknowledge the efforts of the University of Louisville Pneumonia Study Group, University of Louisville, Louisville, KY.

Received: June 18, 2020

Accepted: December 8, 2021

Published: February 17, 2021

Copyright: (c) 2022 The author(s). This original article is brought to you for free and open access by ThinkIR: The Uni- versity of Louisville's Institutional Repository. For more information, please contact thinkir@louisville.edu. This article is distributed under the terms of the Creative Commons Attribution 4.0 International License (CC BY 4.0), which permits unrestricted use, distribution, and reproduction in any medium, provided the original author and source are credited.

Funding Source: The author(s) received no specific funding for this work.

Conflict of Interest: All authors declared no conflict of interest in relation to the main objective of this work.

\section{References}

1. Que Y-A, Moreillon P. Staphylococcus aureus (including staphylococcal toxic shock syndrome). In: Bennett JE, Dolin R, Blaser MJ. Mandell, Douglas, and Bennett's principles and practice of infectious diseases. Eighth ed. Philadelphia, PA: W.B. Saunders, 2015:2237-71.e5.

2. Self WH, Wunderink RG, Williams DJ, et al. Staphylococcus aureus community-acquired pneumonia: Prevalence, clinical characteristics, and outcomes. Clin Infect Dis 2016; 63(3):300-9. doi: 10.1093/cid/ciw300. PMID: 27161775.

3. De la Calle C, Morata L, Cobos-Trigueros N, et al. Staphylococcus aureus bacteremic pneumonia. Eur J Clin Microbiol Infect Dis 2016; 35(3):497-502. doi: 10.1007/s10096-0152566-8. PMID: 26780692.

4. González C, Rubio M, Romero-Vivas J, González M, Picazo JJ. Bacteremic pneumonia due to Staphylococcus aureus: A comparison of disease caused by methicillin-resistant and methicillin-susceptible organisms. Clin Infect Dis 1999; 29(5):1171-7. doi: 10.1086/313440. PMID: 10524959.
5. Metlay JP, Waterer GW, Long AC, et al. Diagnosis and treatment of adults with community-acquired pneumonia: An official clinical practice guideline of the American Thoracic Society and Infectious Diseases Society of America. Am J Respir Crit Care Med 2019; 200(7):e45-e67. doi: 10.1164/rccm.201908-1581ST. PMID: 31573350.

6. Liu C, Bayer A, Cosgrove SE, et al. Clinical practice guidelines by the Infectious Diseases Society of America for the treatment of methicillin-resistant Staphylococcus aureus infections in adults and children. Clin Infect Dis 2011; 52(3):e18-55. doi: 10.1093/cid/ciq146. PMID: 21208910.

7. Ramirez JA, File T, Musher D. Duration of antibiotic therapy for patients with bacteremic Staphylococcus aureus comunity-acquired pneumonia. Univ Louisville J Respir Infect 2018; 2(1):Article 1. doi: 10.18297/jri/vol2/iss1/1.

8. Haessler S, Mackenzie T, Kirkland KB. Long-term outcomes following infection with meticillin-resistant or meticillinsusceptible Staphylococcus aureus. J Hosp Infect 2008; 
69(1):39-45. doi: 10.1016/j.jhin.2008.01.008. PMID: 19745684.

18353493.

9. Lahey T, Shah R, Gittzus J, Schwartzman J, Kirkland K. Infectious diseases consultation lowers mortality from Staphylococcus aureus bacteremia. Medicine (Baltimore) 2009; 88(5):263-7. doi: 10.1097/MD.0b013e3181b8fccb. PMID:
10. Guimaraes AO, Cao Y, Hong K, et al. A prognostic model of persistent bacteremia and mortality in complicated Staphylococcus aureus bloodstream infection. Clin Infect Dis 2019; 68(9):1502-11. doi: 10.1093/cid/ciy739. PMID: 30165412. 